Frank Klinker $•$ Christoph Reineke

\title{
On the characterization of the degree of interpolation polynomials in terms of certain combinatorical matrices
}

Received: 14 February 2018 / Accepted: 4 June 2018 / Published online: 14 June 2018

(C) The Author(s) 2018

Abstract In this note, we show that the degree of the interpolation polynomial for equidistant base points is characterized by the regularity of matrices of combinatorical type.

Mathematics Subject Classification $65 \mathrm{D} 05 \cdot 15 \mathrm{~B} 36 \cdot 15 \mathrm{~A} 15$

المللخص

في هذه الملحوظة، نبيّن أن درجة كثيرات حدود الاستكمال لنقاط أساس متساوية البعد تتميّز بواسطة الانتظام

لمصفوفات توفيقية.

\section{Statement of the main result}

Let $A_{s, \mathbf{a}} \in M_{\ell+1} \mathbb{R}$ be the matrix depending on a vector $\mathbf{a}=\left(a_{0}, \ldots, a_{\ell}\right) \in \mathbb{R}^{\ell+1}$ and an integer $s \geq 0$ and defined by

$$
A_{s, \mathbf{a}}:=\left(\begin{array}{cccc}
1^{\ell-1} & 2^{\ell-1} & \cdots & (\ell+1)^{\ell-1} \\
(\ell+2)^{\ell-1} & (\ell+3)^{\ell-1} & \cdots & (2 \ell+2)^{\ell-1} \\
\vdots & \vdots & & \vdots \\
\left(\ell^{2}\right)^{\ell-1} & \left(\ell^{2}+1\right)^{\ell-1} & \cdots & \left(\ell^{2}+\ell\right)^{\ell-1} \\
0^{s} a_{0} & 1^{s} a_{1} & \cdots & \ell^{s} a_{\ell} .
\end{array}\right) .
$$

Furthermore, denote by $q_{\mathbf{a}}$ the interpolation polynomial of degree at most $\ell$ defined by the base points $x_{0}, \ldots, x_{\ell}$ and the value vector $\mathbf{a}$ :

$$
q_{\mathbf{a}}\left(x_{i}\right)=a_{i}, \quad i=0, \ldots, \ell .
$$

In this text, we show the following remarkable characterization of the degree of the interpolation polynomial for equidistant base points in terms of the matrices $A_{s, \mathbf{a}}$ :

F. Klinker $(\varangle) \cdot$ C. Reineke

Faculty of Mathematics, TU Dortmund University, 44221 Dortmund, Germany

E-mail: frank.klinker@math.tu-dortmund.de

C. Reineke

E-mail: christoph_reineke@gmx.de 
Theorem 1.1 The interpolation problem $q_{\mathbf{a}}\left(x_{i}\right)=a_{i}$ associated with equidistant base points $x_{i}=\xi+i h, i=$ $0,1, \ldots, \ell$ and the value vector $\mathbf{a}=\left(a_{0}, a_{1}, \ldots, a_{\ell}\right)$ yields a polynomial of degree $\ell-m$ if and only if $\operatorname{det} A_{s, \mathbf{a}}=0$, for $s=0, \ldots, m-1$, and $\operatorname{det} A_{m, \mathbf{a}} \neq 0$.

When we were preparing this text, we learned much about the relation between combinatorics and binomial identities and the evaluation of special determinants. We cordially refer the reader to the outstanding collections $[8,9]$ of determinants evaluation. We also refer to the book [11] which presents special combinatorical identities.

\section{The proof of the main Theorem}

Theorem 1.1 relies on some properties of the matrix $A_{s, \mathbf{a}}$ and the polynomial $q_{\mathbf{a}}$ that can be obtained independently from each other. In this section, we state these results and show how they prove Theorem 1.1.

The first result is on the determinant of the matrix (1):

Proposition 2.1 There is a constant $\sigma_{\ell}$ only depending on the size of the matrix $A_{s, \mathbf{a}}$ defined by (1), such that its determinant is given by

$$
\operatorname{det}\left(A_{s, \mathbf{a}}\right)=\sigma_{\ell} \sum_{j=0}^{\ell}(-1)^{j}\left(\begin{array}{l}
\ell \\
j
\end{array}\right) j^{s} a_{j}
$$

The second result deals with the derivatives of the interpolation polynomial (2):

Proposition 2.2 For integers $s \geq 0$ and $0 \leq k \leq s$, there are constants $\sigma_{\ell, s, k}$, such that the $(\ell-s)$ th derivative of the interpolation polynomial (2) for equidistant base points is given by

$$
q_{\mathbf{a}}^{(\ell-s)}(\xi)=h^{s-\ell} \sum_{k=0}^{s} \sigma_{\ell, s, k}\left(\sum_{j=0}^{\ell}(-1)^{j}\left(\begin{array}{l}
\ell \\
j
\end{array}\right) j^{k} a_{j}\right) .
$$

Theorem 1.1 is a corollary of Propositions 2.1 and 2.2:

Proof (Theorem 1.1) The interpolation polynomial $q_{\mathbf{a}}(x)=\sum_{k=0}^{\ell} b_{k}(x-\xi)^{k}$ has degree $\ell-m$ if and only if $b_{\ell}=\cdots=b_{\ell-m+1}=0$ and $b_{\ell-m} \neq 0$. This is equivalent to have vanishing derivatives $q_{\mathbf{a}}^{(\ell-s)}(\xi)$ at the point $x=\xi$ for exactly $s=0, \ldots, m-1$. By (4) with (3) this is equivalent to $\operatorname{det}\left(A_{s, \mathbf{a}}\right)=0$ for $s=0, \ldots, m-1$ and $\operatorname{det}\left(A_{m, \mathbf{a}}\right) \neq 0$.

In the next sections, we prove Propositions 2.1 and 2.2.

\section{On the determinant of $A_{s, \text { a }}$}

\subsection{Preliminaries}

Some submatrices of $A_{s, \mathbf{a}}$ (1) are of special type and were studied in our previous work [7]. They will be important in the reasoning of Proposition 2.1.

In [7], we considered a positive integer $\ell$ and three sequences of complex numbers $\boldsymbol{\alpha}=\left(\alpha_{1}, \alpha_{2}, \ldots\right), \boldsymbol{\beta}=$ $\left(\beta_{1}, \beta_{2}, \ldots\right)$, and $\mathbf{r}=\left(r_{1}, r_{2}, \ldots\right)$. We assume $\mathbf{r}$ to be injective, i.e., $r_{i} \neq r_{j}$ for all $i, j \in \mathbb{N}$. For each $k \in \mathbb{N}$, these data define a complex $k \times k$-matrix $B:=B(k ; \boldsymbol{\alpha}, \boldsymbol{\beta}, \mathbf{r}, \ell) \in M_{k} \mathbb{C}$ :

$$
B_{i j}=\left(\alpha_{i}+r_{j} \beta_{i}\right)^{\ell-1} \text {. }
$$

The determinant of this matrix is $\operatorname{det}(B)=0$ for $k>\ell$. For non vanishing $\alpha_{i}, \beta_{i}$ and $\rho_{i}:=\frac{\beta_{i}}{\alpha_{i}}$, we obtain

$$
\operatorname{det}(B)=\prod_{i=1}^{k} \alpha_{i}^{\ell-1} \sum_{0 \leq \mu_{1}<\cdots<\mu_{k} \leq \ell-1} \prod_{j=1}^{k}\left(\begin{array}{c}
\ell-1 \\
\mu_{j}
\end{array}\right) V_{k, \mu}\left(r_{1}, \ldots, r_{k}\right) V_{k, \mu}\left(\rho_{1}, \ldots, \rho_{k}\right)
$$

or 


$$
\operatorname{det}(B)=(-1)^{\frac{k(k-1)}{2}} \prod_{i=1}^{k} \beta_{i}^{\ell-1} \sum_{0 \leq \mu_{1}<\cdots<\mu_{k} \leq \ell-1} \prod_{j=1}^{k}\left(\begin{array}{c}
\ell-1 \\
\mu_{j}
\end{array}\right) V_{k, \mu}\left(r_{1}, \ldots, r_{k}\right) V_{k, \mu^{\mathrm{C}}}\left(\frac{1}{\rho_{1}}, \ldots, \frac{1}{\rho_{k}}\right)
$$

for $k \leq \ell$, where $\mu$ is a sequence $\mu=\left(\mu_{1}, \ldots, \mu_{k}\right)$ of strictly increasing natural numbers $0 \leq \mu_{1}<\cdots<$ $\mu_{k} \leq \ell-1, \mu^{\complement}$ is the associated strictly increasing sequence $\mu^{\complement}=\left(\ell-1-\mu_{k}, \ldots, \ell-1-\mu_{1}\right)$, and

$$
V_{k, \mu}\left(v_{1}, \ldots, v_{k}\right)=\operatorname{det}\left(v_{i}^{\mu_{j}}\right)
$$

denotes the generalized Vandermonde determinant, see [5]. In particular, $V_{k,(0,1, \ldots, k-1)}=V_{k}$, where $V_{k}$ is the usual Vandermonde determinant with $V_{k}\left(v_{1}, \ldots, v_{k}\right)=\prod_{1 \leq i<j \leq k}\left(v_{j}-v_{i}\right)$. Since $V_{k, \mu}\left(v_{1}, \ldots v_{k}\right)$ vanishes whenever $v_{i}=v_{j}$ the quotient $V_{k, \mu} / V_{k}$ is a polynomial, too. These symmetric polynomials are the Schur polynomials, see, e.g., [1,6,10].

A consequence of this discussion is

Theorem 3.1 ([7, Theorem 3]) Let $\boldsymbol{\alpha}, \boldsymbol{\beta}$ be sequences of complex numbers with ${ }^{1} \frac{\alpha_{i}}{\beta_{i}} \in \mathbb{R}^{+}$and $\alpha_{i} \beta_{j}-\beta_{i} \alpha_{j} \neq 0$ for all $1 \leq i, j \leq k$, and $\mathbf{r}$ be a positive injective sequence. Then, the matrix (5) is regular if and only if $k \leq \ell$.

3.2 The matrix $A_{s, \mathbf{a}}$ and its determinant

Let us consider the matrix $A_{s, \mathbf{a}} \in M_{\ell+1} \mathbb{R}$ defined by (1). Its components are given by ${ }^{2}$

$$
\begin{aligned}
& \left(A_{s, \mathbf{a}}\right)_{i j}= \begin{cases}\left(\alpha_{i}+r_{j} \beta_{i}\right)^{\ell-1} & \text { for } 1 \leq i \leq \ell, 1 \leq j \leq \ell+1 \\
(j-1)^{s} a_{j-1} & \text { for } i=\ell+1,1 \leq j \leq \ell+1\end{cases} \\
& \text { with } \alpha_{i}:=(i-1)(\ell+1), \quad \beta_{i}=1, \quad r_{j}=j .
\end{aligned}
$$

Furthermore, denote by $A^{[\kappa]} \in M_{\ell} \mathbb{R}$ the matrix obtained from $A_{s, \mathbf{a}}$ by removing the last row and the $\kappa$ th column. Of course, it does not depend on $s$ and a and is given by

$$
\begin{aligned}
& \left(A^{[\kappa]}\right)_{i j}=\left(\alpha_{i}+r_{j}^{[\kappa]} \beta_{i}\right)^{\ell-1} \\
& \text { with } r_{j}^{[\kappa]}= \begin{cases}j & \text { for } 1 \leq j \leq \kappa-1, \\
j+1 & \text { for } \kappa \leq j \leq \ell .\end{cases}
\end{aligned}
$$

The matrices $A^{[\kappa]}$ are of the form (5) and have the following property:

Proposition 3.1 There exist a number $\sigma_{\ell}$ depending only on $\ell$ such that

$$
\operatorname{det}\left(A^{[\kappa]}\right)=(-1)^{\ell} \sigma_{\ell}\left(\begin{array}{c}
\ell \\
\kappa-1
\end{array}\right) \text {. }
$$

An immediate corollary is Proposition 2.1:

Proof (Proposition 2.1) Expanding the determinant of $A_{s, \mathbf{a}}$ with respect to the last row yields

$$
\begin{aligned}
\operatorname{det}\left(A_{s, \mathbf{a}}\right) & =\sum_{j=1}^{\ell+1}(-1)^{\ell+1+j} A_{\ell+1, j} \operatorname{det} A^{[j]} \\
& =(-1)^{\ell} \sum_{j=0}^{\ell}(-1)^{j} j^{s} a_{j} \operatorname{det} A^{[j+1]} \\
& =\sigma_{\ell} \sum_{j=0}^{\ell}(-1)^{j}\left(\begin{array}{l}
\ell \\
j
\end{array}\right) j^{s} a_{j} .
\end{aligned}
$$

${ }^{1}$ In [7] we show that the regularity result remains valid when one of the $\alpha_{i}$ or $\beta_{i}$ vanishes.

${ }^{2}$ We use the convention $0^{0}=1$ to cover all cases by this formula. 
In the sequel, we prove Proposition 3.1:

Proof (Proposition 3.1) We use (6') for $k=\ell$ and note that only one summand is left, namely, the one with $\mu=\mu^{\complement}=(0,1, \ldots \ell-1)$. In this case, we have $V_{\ell, \mu}=V_{\ell, \mu^{\complement}}=V_{\ell}$, and therefore,

$$
\begin{aligned}
\operatorname{det}\left(A^{[\kappa]}\right) & =(-1)^{\frac{\ell(\ell-1)}{2}} \prod_{i=1}^{\ell} \beta_{i}^{\ell-1} \prod_{j=1}^{\ell}\left(\begin{array}{c}
\ell-1 \\
j-1
\end{array}\right) V_{\ell}\left(\frac{\alpha_{1}}{\beta_{1}}, \ldots, \frac{\alpha_{\ell}}{\beta_{\ell}}\right) V_{\ell}\left(r_{1}^{[\kappa]}, \ldots, r_{\ell}^{[\kappa]}\right) \\
& =(-1)^{\frac{\ell(\ell-1)}{2}} \prod_{j=0}^{\ell-1}\left(\begin{array}{c}
\ell-1 \\
j
\end{array}\right) \prod_{1 \leq j_{1}<j_{2} \leq \ell}\left(\alpha_{j_{2}}-\alpha_{j_{1}}\right) \prod_{1 \leq i_{1}<i_{2} \leq \ell}\left(r_{i_{2}}^{[\kappa]}-r_{i_{1}}^{[\kappa]}\right) \\
& =(-1)^{\frac{\ell(\ell-1)}{2}}(\ell+1)^{\frac{\ell(\ell-1)}{2}} \prod_{j=0}^{\ell-1}\left(\begin{array}{c}
\ell-1 \\
j
\end{array}\right) \prod_{1 \leq j_{1}<j_{2} \leq \ell}\left(j_{2}-j_{1}\right) \prod_{1 \leq i_{1}<i_{2} \leq \ell}\left(r_{i_{2}}^{[\kappa]}-r_{i_{1}}^{[\kappa]}\right) \\
& =(-1)^{\frac{\ell(\ell-1)}{2}}(\ell+1)^{\frac{\ell(\ell-1)}{2}} \prod_{j=0}^{\ell-1}\left(\begin{array}{c}
\ell-1 \\
j
\end{array}\right) \prod_{j=1}^{\ell-1} j ! \prod_{1 \leq i_{1}<i_{2} \leq \ell}\left(r_{i_{2}}^{[\kappa]}-r_{i_{1}}^{[\kappa]}\right) .
\end{aligned}
$$

The proof is completed if we show that the quotient of $\prod_{1 \leq i_{1}<i_{2} \leq \ell}\left(r_{i_{2}}^{[\kappa]}-r_{i_{1}}^{[\kappa]}\right)$ and $\left(\begin{array}{c}\ell \\ \kappa-1\end{array}\right)$ only depends on $\ell$. For $j>i$, we have

$$
r_{j}^{[\kappa]}-r_{i}^{[\kappa]}= \begin{cases}j-i & \text { if } 1 \leq i<j \leq \kappa-1 \text { or } \kappa \leq i<j \leq \ell \\ j-i+1 & \text { if } 1 \leq i \leq \kappa-1, \kappa \leq j \leq \ell\end{cases}
$$

and therefore

$$
\begin{aligned}
\prod_{1 \leq i_{1}<i_{2} \leq \ell}\left(r_{i_{2}}^{[\kappa]}-r_{i_{1}}^{[\kappa]}\right) & =\left(\prod_{j=1}^{\kappa-1} \prod_{i=1}^{j-1}(j-i)\right)\left(\prod_{j=\kappa}^{\ell} \prod_{i=\kappa}^{j-1}(j-i)\right)\left(\prod_{j=\kappa}^{\ell} \prod_{i=1}^{\kappa-1}(j-i+1)\right) \\
& =\prod_{i_{1}=1}^{\kappa-2} i_{1} ! \prod_{i_{2}=1}^{\ell-\kappa} i_{2} ! \prod_{j=\kappa}^{\ell} \prod_{i=1}^{\kappa-1}(j-i+1) \\
& =\prod_{i_{1}=1}^{\kappa-2} i_{1} ! \prod_{i_{2}=1}^{\ell-\kappa} i_{2} ! \prod_{m=1}^{\ell-\kappa+1} \prod_{n=1}^{\kappa-1}(m+n) \\
& =\prod_{i_{1}=1}^{\kappa-2} i_{1} ! \prod_{i_{2}=1}^{\ell-\kappa} i_{2} ! \prod_{p=2}^{\ell} p^{\gamma_{p}}
\end{aligned}
$$

Here, $\gamma_{p}$ denotes the number of factors with value $p$ in the third product, that is

$$
\gamma_{p}= \begin{cases}p-1 & 2 \leq p \leq \kappa \\ \kappa-1 & \kappa+1 \leq p \leq \ell-\kappa+1 \\ \ell-p+1 & \ell-\kappa+2 \leq p \leq \ell\end{cases}
$$

Rearranging the factors, we obtain

$$
\begin{aligned}
\prod_{1 \leq i_{1}<i_{2} \leq \ell}\left(r_{i_{2}}^{[\kappa]}-r_{i_{1}}^{[\kappa]}\right) & =\prod_{i_{1}=1}^{\kappa-2} i_{1} ! \prod_{i_{2}=1}^{\ell-\kappa} i_{2} ! \prod_{q=1}^{\kappa-1} \frac{(\ell-q+1) !}{q !} \\
& =\prod_{i_{1}=1}^{\kappa-2} i_{1} ! \prod_{i_{3}=1}^{\kappa-1} \frac{1}{i_{3} !} \prod_{i_{2}=1}^{\ell-\kappa} i_{2} ! \cdot \prod_{i_{4}=\ell-\kappa+2}^{\ell} i_{4} ! \\
& =\frac{1}{(\kappa-1) !} \prod_{i_{2}=1}^{\ell-\kappa} i_{2} ! \prod_{i_{4}=\ell-\kappa+2}^{\ell} i_{4} !
\end{aligned}
$$




$$
\begin{aligned}
& =\frac{1}{(\kappa-1) !} \frac{\ell !}{(\ell-\kappa+1) !} \prod_{j=1}^{\ell-1} j ! \\
& =\left(\begin{array}{c}
\ell \\
\kappa-1
\end{array}\right) \prod_{j=1}^{\ell-1} j ! .
\end{aligned}
$$

We write

$$
\sigma_{\ell}:=(-1)^{\frac{\ell(\ell+1)}{2}}(\ell+1)^{\frac{\ell(\ell-1)}{2}} \prod_{j=0}^{\ell-1}\left(\begin{array}{c}
\ell-1 \\
j
\end{array}\right) \prod_{j=1}^{\ell-1}(j !)^{2}
$$

and finally achieve

$$
\operatorname{det}\left(A^{[\kappa]}\right)=(-1)^{\ell} \sigma_{\ell}\left(\begin{array}{c}
\ell \\
\kappa-1
\end{array}\right)
$$

Remark 3.1 The proof of Proposition 3.1 can also be performed by reducing the determinant at hand to a matrix with polynomial entries:

$$
p_{j}^{[\kappa]}(i)=a_{j}^{[\kappa]} i^{j-1}+\text { lower order terms } \quad \text { or } \quad p_{i}\left(r_{j}^{[\kappa]}\right)=a_{i}\left(r_{j}^{[\kappa]}\right)^{i-1}+\text { lower order terms. }
$$

Then, applying the determinant evaluation as known from the Vandermonde matrix, one can show that the determinant is given by

$$
\left(\prod_{j=1}^{\ell-1} a_{j}^{[\kappa]}\right) V_{\ell}(1, \ldots, \ell) \quad \text { or } \quad\left(\prod_{i=1}^{\ell-1} a_{i}\right) V_{\ell}\left(r_{1}^{[\kappa]}, \ldots, r_{\ell}^{[\kappa]}\right),
$$

see [8, Propostion 1]. However, in both cases, the explicit calculations above remain to be done.

\section{On the interpolant $q_{\mathrm{a}}$}

In this section, we study the interpolation polynomial $q_{\mathbf{a}}(2)$ associated with equidistant base points and a value vector a, see, e.g., [3,4].

Explicitly, we have

$$
q_{\mathbf{a}}(x)=\sum_{j=0}^{\ell} a_{j} L_{j}(x)
$$

where $L_{j}$ denotes the $j$ th Lagrange polynomial associated with the base points $x_{0}=\xi, x_{1}=\xi+h, \ldots, x_{\ell}=$ $\xi+\ell h$. For $j=0, \ldots, \ell$, the latter are given by

$$
L_{j}(x)=\frac{\prod_{i=0, i \neq j}^{\ell}\left(x-x_{i}\right)}{\prod_{i=0, i \neq j}^{\ell}\left(x_{j}-x_{i}\right)} .
$$

The proof of Proposition 2.2 will be presented at the end of this section and it makes use of a combinatorical fact on the polynomial:

$$
K(t)=\prod_{i=0}^{\ell}(t-i)
$$


To formulate this fact, we introduce the symbols:

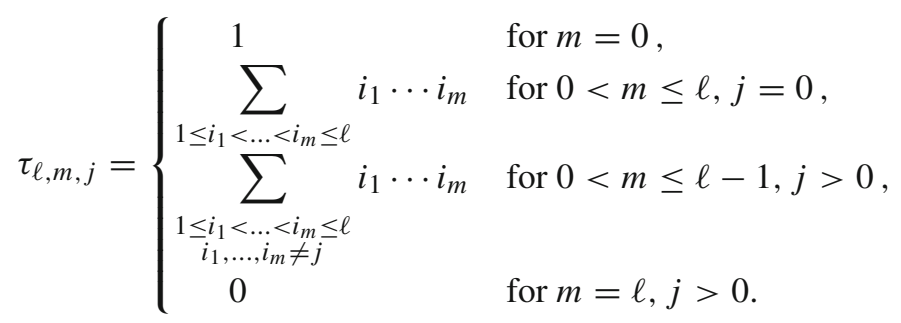

For instance, $\tau_{\ell, 1, j}=\frac{\ell(\ell+1)}{2}-j$ for $0 \leq j \leq \ell$ and $\tau_{\ell, \ell, 0}=\ell !, \tau_{\ell, \ell-1, j}=\frac{\ell !}{j}$ for $1 \leq j \leq \ell$.

Proposition 4.1 For $0 \leq j \leq \ell$, the polynomial $K(t)$ of degree $\ell+1$ obeys

$$
K(t)=(t-j) \sum_{m=0}^{\ell}(-1)^{m} \tau_{\ell, m, j} t^{\ell-m} .
$$

Furthermore, for $j>0$ and $m \neq \ell$, the symbols $\tau_{\ell, m, j}$ obey

$$
\tau_{\ell, m, j}=\sum_{k=0}^{m}(-1)^{k} \tau_{\ell, m-k, 0} j^{k}
$$

Proof The first part is obtained by expanding of $\frac{K(t)}{t-j}=\prod_{\substack{i=0 \\ i \neq j}}^{\ell}(t-i)$. The second part is obviously true for $j=0$. For $j>0$, we note

$$
\begin{aligned}
& \tau_{\ell, m, j}=\sum_{\substack{1 \leq i_{1}<\ldots<i_{m} \leq \ell \\
i_{1}, \ldots, i_{m} \neq j}} i_{1} \cdots i_{m} \\
& =\sum_{1 \leq i_{1}<\ldots<i_{m} \leq \ell} i_{1} \cdots i_{m}-j \sum_{k=1}^{m} \sum_{\substack{1 \leq i_{1}<\ldots<i_{m} \leq \ell \\
i_{k}=j}} i_{1} \cdots i_{k-1} i_{k+1} \cdots i_{m} \\
& =\tau_{\ell, m, 0}-j \sum_{k=1}^{m} \sum_{\substack{1 \leq i_{1}<\ldots<i_{k-1}<j \\
j<i_{k+1}<\ldots<i_{m} \leq \ell}} i_{1} \cdots i_{k-1} i_{k+1} \cdots i_{m} \\
& =\tau_{\ell, m, 0}-j \sum_{k=1}^{m} \sum_{\substack{1 \leq \bar{i}_{1}<\ldots<\bar{i}_{k-1}<j \\
j<\bar{i}_{k}<\ldots<\bar{i}_{m-1} \leq \ell}} \bar{i}_{1} \ldots \bar{i}_{m-1} \\
& =\tau_{\ell, m, 0}-j \sum_{\substack{1 \leq \bar{i}_{1}<\ldots<\bar{i}_{m-1} \leq \ell \\
\bar{i}_{1}, \ldots, \bar{i}_{m-1} \neq j}} \bar{i}_{1} \ldots \bar{i}_{m-1} \\
& =\tau_{\ell, m, 0}-j \tau_{\ell, m-1, j} \text {. }
\end{aligned}
$$

Using this recurrence, (12) holds by induction.

Proof (Proposition 2.2)

We define $x=\xi+t h, \hat{L}_{j}(t):=L_{j}(\xi+t h)$, and $\hat{q}_{\mathbf{a}}(t):=q_{\mathbf{a}}(\xi+t h)$, that is

$$
\hat{L}_{j}(t)=\frac{\prod_{i=0, i \neq j}^{\ell} h(t-i)}{\prod_{i=0, i \neq j}^{\ell} h(j-i)}=\frac{\prod_{i=0, i \neq j}^{\ell}(t-i)}{\prod_{i=0, i \neq j}^{\ell}(j-i)}=\frac{(-1)^{\ell-j}}{\ell !}\left(\begin{array}{l}
\ell \\
j
\end{array}\right) \frac{K(t)}{t-j}
$$


and

$$
\hat{q}_{\mathbf{a}}(t)=\sum_{j=0}^{\ell} a_{j} \hat{L}_{j}(t)=\frac{(-1)^{\ell}}{\ell !} \sum_{j=0}^{\ell}(-1)^{j}\left(\begin{array}{l}
\ell \\
j
\end{array}\right) a_{j} \frac{K(t)}{t-j}
$$

with $K(t)$ defined in (10). By Proposition 4.1, we have

$$
\hat{q}_{\mathbf{a}}(t)=\frac{(-1)^{\ell}}{\ell !} \sum_{m=0}^{\ell} \sum_{k=0}^{m}(-1)^{k+m} \tau_{\ell, m-k, 0}\left(\sum_{j=0}^{\ell}(-1)^{j}\left(\begin{array}{l}
\ell \\
j
\end{array}\right) j^{k} a_{j}\right) t^{\ell-m}
$$

and

$$
\hat{q}_{\mathbf{a}}^{(\ell-s)}(t)=\frac{(-1)^{\ell}(\ell-s) !}{\ell !} \sum_{m=0}^{s}\left(\begin{array}{l}
\ell-m \\
s-m
\end{array}\right) \sum_{k=0}^{m}(-1)^{k+m} \tau_{\ell, m-k, 0}\left(\sum_{j=0}^{\ell}(-1)^{j}\left(\begin{array}{l}
\ell \\
j
\end{array}\right) j^{k} a_{j}\right) t^{s-m} .
$$

In particular, we obtain at $t=0$

$$
\frac{(-1)^{\ell-s} \ell !}{(\ell-s) !} \hat{q}_{\mathbf{a}}^{(\ell-s)}(0)=\sum_{k=0}^{s}(-1)^{k} \tau_{\ell, s-k, 0}\left(\sum_{j=0}^{\ell}(-1)^{j}\left(\begin{array}{l}
\ell \\
j
\end{array}\right) j^{k} a_{j}\right) .
$$

For instance, for the first values of $s$, the right hand side of this expression is

$$
\begin{aligned}
& s=0: \tau_{\ell, 0,0} \sum_{j=0}^{\ell}(-1)^{j}\left(\begin{array}{l}
\ell \\
j
\end{array}\right) a_{j}, \\
& s=1: \tau_{\ell, 1,0} \sum_{j=0}^{\ell}(-1)^{j}\left(\begin{array}{l}
\ell \\
j
\end{array}\right) a_{j}-\tau_{\ell, 0,0} \sum_{j=0}^{\ell}(-1)^{j}\left(\begin{array}{l}
\ell \\
j
\end{array}\right) j a_{j}, \\
& s=2: \tau_{\ell, 2,0} \sum_{j=0}^{\ell}(-1)^{j}\left(\begin{array}{l}
\ell \\
j
\end{array}\right) a_{j}-\tau_{\ell, 1,0} \sum_{j=0}^{\ell}(-1)^{j}\left(\begin{array}{l}
\ell \\
j
\end{array}\right) j a_{j}+\tau_{\ell, 0,0} \sum_{j=0}^{\ell}(-1)^{j}\left(\begin{array}{l}
\ell \\
j
\end{array}\right) j^{2} a_{j} .
\end{aligned}
$$

Writing

$$
\sigma_{\ell, s, k}:=\frac{(-1)^{\ell-s+k}(\ell-s) !}{\ell !} \tau_{\ell, s-k, 0}
$$

and using $\hat{q}_{\mathbf{a}}^{(\ell-s)}(\xi+t h)=h^{\ell-s} q_{\mathbf{a}}^{(\ell-s)}(t)$, we obtain (4).

\section{A final remark on the general interpolation problem}

Remark 5.1 In the case of arbitrary base points $x_{0}, \ldots, x_{\ell}$, one can introduce modified symbols $\tau_{\ell, m, j}=$ $\sum_{\substack{1 \leq i_{1}<\ldots, i_{m} \leq \ell \\ i_{1}, \ldots, i_{m} \neq j}} x_{i_{1}} \cdots x_{i_{m}}$ to get an analogous of (11).

In [2], these symbols are denoted by $\widehat{e}_{\ell-m, j}$ and are used to present representations of the Schur functions. In our setting, they can be used to get an expansion like (12), too, and a formula of the form (13) can also be obtained:

$$
q_{\mathbf{a}}(x)=\sum_{m=0}^{\ell} \sum_{k=0}^{m}(-1)^{k+m} \tau_{\ell, m-k, 0}\left(\sum_{j=0}^{\ell} \lambda_{j} x_{j}^{k} a_{j}\right) x^{\ell-m}
$$

with $\lambda_{j}^{-1}=\prod_{\substack{i=0 \\ i \neq j}}^{\ell}\left(x_{i}-x_{j}\right)$. However, a result as in Theorem 1.1 is not obtained in such a nice form.

Open Access This article is distributed under the terms of the Creative Commons Attribution 4.0 International License (http:// creativecommons.org/licenses/by/4.0/), which permits unrestricted use, distribution, and reproduction in any medium, provided you give appropriate credit to the original author(s) and the source, provide a link to the Creative Commons license, and indicate if changes were made. 


\section{References}

1. De Marchi, S.: Polynomials arising in factoring generalized Vandermonde determinants: an algorithm for computing their coefficients. Math. Comput. Model. 34(3/4), 271-281 (2001)

2. de Camargo, A.P.: Schur functions through Lagrange polynomials. J. Pure Appl. Algebra 220(8), 2948-2954 (2016)

3. Davis, P.J.: Interpolation and Approximation. Dover Publication Inc, New York (1975)

4. Deuflhard, P.; Hohmann, A.: Numerical Analysis in Modern Scientific Computing. Springer, New York (2003)

5. Heineman, E.R.: Generalized Vandermonde determinants. Trans. Am. Math. Soc. 31(3), 464-476 (1929)

6. King, R.C.: Generalised Vandermonde determinants and Schur functions. Proc. Am. Math. Soc. 48(1), 53-56 (1975)

7. Klinker, F., Reineke, C.: On the regularity of matrices with uniform polynomial entries. Sao Paulo J. Math. Sci. (2018), online 2017

8. Krattenthaler, C.: Advanced determinant calculus. Sem. Lothar. Combin. 42 Art. B42q, 67 (1999)

9. Krattenthaler, C.: Advanced determinant calculus: a complement. Linear Algebra Appl. 411, 68-166 (2005)

10. Littlewood, D.E.: The Theory of Group Characters and Matrix Representations of Groups. Oxford University Press, New York (1940)

11. Riordan, J.: Combinatorical Identities. Reprint of the 1968 Original. Robert E. Krieger Publishing Co., Huntington (1979)

Publisher's Note Springer Nature remains neutral with regard to jurisdictional claims in published maps and institutional affiliations. 